\title{
K-Band Spectroscopy of (Pre-)Cataclysmic Variables: Are Some Donor Stars Really Carbon Poor?
}

\author{
Steve B. Howell ${ }^{1,4}$ \\ Thomas E. Harrison ${ }^{2,4}$ \\ Paula Szkody $y^{3,4}$ \\ and \\ Nicole M. Silvestri ${ }^{3}$
}

Received —

\footnotetext{
${ }^{1}$ NOAO, 950 N. Cherry Ave., Tucson, AZ 85719: (E-mail: howell@noao.edu)

${ }^{2}$ New Mexico State University, P.O. Box 30001, Las Cruces, NM 88003: (E-mail: tharriso@nmsu.edu)

${ }^{3}$ University of Washington: (E-mail: nms/szkody@washington.astro.edu)

${ }^{4}$ Visiting observer Keck II telescope
} 


\begin{abstract}
We present a new sample of $K$-band spectral observations for CVs: non-magnetic and magnetic as well as present day and pre CVs. The purpose of this diverse sample is to address the recent claim that the secondary stars in dwarf novae are carbon deficient, having become so through a far more evolved evolution than the current paradigm predicts. Our new observations, along with previous literature results, span a wide range of orbital period and CV type. In general, dwarf novae in which the secondary star is seen show weak to no $\mathrm{CO}$ absorption while polar and pre-CV donor stars appear to have normal $\mathrm{CO}$ absorption for their spectral type. However, this is not universal. The presence of normal looking CO absorption in the dwarf nova SS Aur and the hibernating CV QS Vir and a complete lack of CO absorption in the long period polar V1309 Ori cloud the issue. A summary of the literature pointing to non-solar abundances including enhanced NV/CIV ratios is presented. It appears that some CVs have non-solar abundance material accreting onto the white dwarf suggesting an evolved secondary star while for others CO emission in the accretion disk may play a role. However, the exact mechanism or combination of factors causing the $\mathrm{CO}$ absorption anomaly in $\mathrm{CVs}$ is not yet clear.
\end{abstract}

Subject headings: cataclysmic variables — stars:individual (V1309 Ori, V471 Tau, QS Vir, BPM 71213, BT Mon, GW Lib, SS Aur, RZ Leo, ST LMi, SDSSJ083038.80+470246.97, SDSSJ075721.06+323054.39, SDSSJ074301.92+410655.18, SDSSJ083751.00+383012.5, HS1136+6646) spectroscopy:stars 


\section{Introduction}

Cataclysmic variables are semi-detached binaries in which a white dwarf primary is accreting material from its close neighbor, a low-mass Roche-lobe filling object. The donor stars are often taken to be of the main sequence variety, but observational evidence often suggests that they are more evolved. Close binary star evolution has some known basic properties (e.g., gravitational radiation) but the details of the "standard model" are not yet fully in hand. Additionally, some "oddball" systems (e.g., Eggleton 2006) are known and generally swept under the rug.

Over the past few years, infrared spectroscopy has revealed a new factor to be dealt with in our understanding of binary evolution - the apparent carbon deficiency or low carbon abundance observed in the secondary stars of some dwarf novae type cataclysmic variables (Harrison et al., 2004). To make things even more interesting, it appears that the weakness of carbon (as evidenced by very weak or missing CO absorption in the spectra) is only mainly an issue for cataclysmic variables (CVs) with non-magnetic white dwarf (WD) primary stars. The highly magnetic WD primary systems, the polars, have secondary stars that appear to be completely normal (Harrison et al., 2005).

The generally accepted present-day paradigm for CV evolution (Howell et al., 2001) cannot account for the low carbon abundance observed or for a different evolutionary sequence for magnetic CVs vs. non-magnetic CVs. Evolution models such as those by Beuermann et al. (1998) and Barraffe and Kolb (2000) explore donor stars which have significantly evolved prior to the start of mass transfer. Marks and Sarna (1998) explore the chemical evolution expected in secondary star systems which undergo nova explosions and which go through a common envelope stage.

Gansicke et al. (2003) note that eight CVs are known to have large NV/CIV emission line ratios as seen in their UV spectra. Cheng et al. (1997) found that the TOAD WZ Sge 
was over abundant in carbon $(5 \mathrm{x})$ as well as in nitrogen $(3 \mathrm{x})$ with respect to solar. Thus, it is not just the weak or absent $\mathrm{CO}$ observed in the $K$-band that seems to be implying that some systems have non-solar abundances, generally indicative of more evolved stars in which CNO burning has occurred. To date, there has been no detailed elemental abundance study of CV donor stars, a useful enterprise to undertake in order to provide metalicity values and evolution indicators such as the $12 \mathrm{C}$ to $13 \mathrm{C}$ ratio.

We present new IR spectroscopy of a number of CVs and related non-interacting pre-CV binaries. Using these new results, along with those previously published in the literature, allows us to span a wide range in orbital period and CV subtype. We can then formulate a picture of the nature of the secondary stars as a function of orbital period, interaction state, and magnetic field of the primary white dwarf. We attempt to use the observations to constrain the carbon abundance as it relates to binary evolution.

\section{Infrared Spectroscopic Observations}

All our infrared spectra presented here were obtained at the Keck II telescope on Mauna Kea, Hawaii on 4 and 5 March 2007 UT 17 using the NIRSPEC. NIRSPEC was used in low resolution mode with the slit width set to 0.38 " as we had seeing near 0.4 " on both nights. We chose a grating tilt to cover the $K$-band spectral range of 2.04-2.40 micron with a dispersion of $4.27 \AA$ /pixel or a velocity resolution near $110 \mathrm{~km} / \mathrm{sec}$. All stars were observed using a standard ABBA nod pattern on the slit and the four nod exposures were combined into final spectra depending on the $\mathrm{S} / \mathrm{N}$ per nod. Our observations were not all obtained under photometric conditions and the use of a narrow slit both preclude assignment of true fluxes to the spectra.

Observations of bright A-type stars nearby in time and location were used for telluric 
correction. Spectrophotometric stars were observed with the same setup and we estimate our fluxes are accurate to $\sim 15 \%$. The Keck spectra were reduced using the IDL routine REDSPEC especially developed for NIRSPEC reductions. A stars are nearly featureless in the $K$-band except for Brackett $\gamma$ which was removed by interpolation across the continuum on both sides of the line during reductions. A complete description of our reduction process is presented in Harrison et al. (2004) and Table 1 presents a log of the IR observations.

\section{Analysis}

We present results for each star separately below, organized by their orbital period; long to short. Late-type dwarf templates, near the observed donor star (or expected donor star) spectral type, are shown in some figures for comparison purposes and all are from

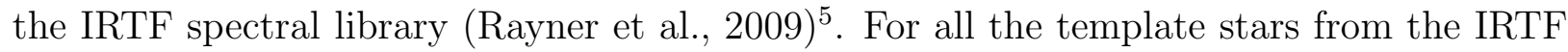
spectral library, fluxes are given in $\mathrm{W} \mathrm{m}^{-2} \mu \mathrm{m}^{-1}$ while our Keck spectra are presented in normalized flux units of the same kind.

For most of the systems listed below, we estimate the spectral type of the secondary star. These spectral types are based on the similarities in strengths of the strongest absorption features to MK template spectra. As with our previous efforts, however, unusual line strengths can be present which confuse the precise classification, and any such spectral types are probably only good to \pm one subtype for the best of the spectra. The emperical method we use is discussed in detail in Harrison et al. (2004, 2005b). Table 2 summarizes our findings for the present data set. We will abbreviate SDSS sources in the paper as follows: SDSS0830 (=SDSSJ083038.80+470246.97), SDSS0757

\footnotetext{
${ }^{5}$ The IRTF spectral library is available in digital form at http://irtfweb.ifa.hawaii.edu/ spex/IRTF_Spectral_Library/index.html.
} 
(=SDSSJ075721.06+323054.39), SDSS0743 (=SDSSJ074301.92+410655.18), and SDSS0837 (=SDSSJ083751.00+383012.5).

\subsection{HS1136+6646}

HS1136+6646 was identified as a young, post common envelope DAO white dwarf binary, that is a pre-CV, by Sing et al. (2004). Velocity analysis of optical spectra of HS1136 revealed a hot WD and narrow emission lines assumed to be from an irradiated secondary. Using this information, Sing et al. inferred a likely M2.5V secondary star in a 20 hour orbit. Later, Liebert et al. (2006) discovered a third body in the system at a separation of 1.3 arcsec with a position angle of $54.5 \mathrm{deg}$, which was likely a common proper motion object.

When we pointed the Keck II telescope at HS1136, the acquisition imager (SCAM) easily revealed two sources differing in brightness by 1.9 mag with the pre-CV (SW component) being fainter than the K7V (NE) component. We measured a separation of 1.2 " at a position angle of $\sim 52$ degrees, consistent with the values found by Liebert et al. (2006).

We obtained a $K$-band spectrum of both sources, the CV and the $\mathrm{K}$ star (Figure 1 ). Narrow emission lines due to He (2.056 microns) and Brackett $\gamma$ are seen in the pre-CV spectrum. Sing et al. (2004) attributed the optical emission lines to irradiation of the secondary (by the 70,000K WD) and noted that only the He II $\lambda 4686$ emission line moved with the white dwarf velocity. However, they found from high resolution spectroscopy that $\mathrm{H} \alpha$ shows a double-peaked structure (velocity separation $=\sim 90 \mathrm{~km} / \mathrm{sec}$ ) with a stronger narrow component, suggestive of the presence of accretion disk emission in addition to the irradiation lines. The HeII $\lambda 4686$ emission line gave $\mathrm{K} 1=69+/-2 \mathrm{~km} / \mathrm{s}$, and an origin from 
the white dwarf was confirmed as this line had similar velocity amplitude and phase to WD lines measured from phase-resolved FUSE observations (Sing et al. 2004).

We see no absorption features from the $\mathrm{M} 2.5 \mathrm{~V}$ secondary in our $K$-band spectrum of the pre-CV. This is not surprising as the hot $(70,000 \mathrm{~K})$ white dwarf continuum dominates

the system flux even into the $K$-band, and the secondary was only observed in the optical due to $\mathrm{H}$ emission lines produced by strong irradiation.

The NE component, the common proper motion K7V star, has an IR spectrum that presents Mg I (2.106 microns), Al I (2.116 microns), Na I (2.21 \& 2.35 microns), Ca I (2.26 microns), and $\mathrm{CO}$ in absorption. The $K$-band spectrum of this object is contaminated by the closeness of the $\mathrm{CV}$, and in particular we see in Fig. 1 some residual $\operatorname{Br} \gamma$ emission from the pre-CV.

\subsection{V471 Tau}

This famous Hyades member binary (Ibanoglu et al., 1994; Rottler et al., 2002; and references therein) was observed as a "standard" pre-CV star. The 0.52 day binary consists of a white dwarf and a K2V secondary star, the latter being highly active. O'Brien et al. (2001) discuss oddities in both stars, the white dwarf being too young and too hot for its mass as a cluster member and the $\mathrm{K} 2 \mathrm{~V}$ dwarf being too large compared with other similar mass K2 dwarfs in the Hyades cluster. V471 Tau is often cited as the canonical pre-CV. Dhillon et al. (2002) refute the earlier claim by Dhillon \& Marsh (1995) and Catalan et al. (2001) that there is anything unusual about the $13 \mathrm{CO}$ feature at 2.345 microns in V471 Tau.

We find that our IR spectrum of V471 Tau agrees well with a K2 \pm 1 main sequence star being similar to the two templates shown in Figure 2. This classification agrees with 
the K2V choice of Dhillon et al. (2002) using the $13 \mathrm{CO}$ feature. We find no absorption features that are inconsistent with normal solar abundances and the CO bands are present and with the strengths expected for an early KV star.

Assuming a 100\% contribution from a normal $\mathrm{K} 2 \mathrm{~V}$ star $\left(\mathrm{M}_{K}=7.3\right)$ and convolving a $\mathrm{K}$ filter with our spectrum (giving $\mathrm{K}=10.6$ ), we find a distance to V471 Tau of 47.4 pc, in agreement with the HIPPARCOS parallax value of 47.6 pc (de Bruijne, Hoogerwerf, \& de Zeeuw, 2001). Other than the slight oddities noted in the literature, the secondary star in V471 Tau appears to be fairly normal.

\subsection{BT Mon}

BT Mon is an old nova (Nova Mon 1939) with an orbital period near $1 / 3$ of a day and with deep (2.7 mag) eclipses. Currently it is classified as a dwarf nova or a novalike (Warner 1995). At 15th magnitude in $\mathrm{V}$, this binary has not been studied extensively but two comprehensive optical spectroscopic works have been presented (White et al., 1996; Smith et al., 1998). White et al. found inconclusive evidence to support an accretion disk in BT Mon but suggested a possible magnetic white dwarf exists in the binary. Smith et al. detected weak absorption star features from the secondary during eclipse. Comparison with template spectra provided them with a best fit at G8V, but this was the same spectral class as the earliest template they used so an earlier type donor star may be possible. Using the relation determined by Smith and Dhillon (1998), BT Mon's secondary star should be near $\mathrm{K} 3 \mathrm{~V}$.

$K$-band spectroscopy, presented in Figure 3, shows weak HeI emission and a strong, narrow Brackett $\gamma$ line. The blue continuum and the strong emission lines argue for a strong disk contribution in BT Mon, a typical result of the high mass transfer rate of old novae. 
Thus, any secondary star contributions are outshone by the accretion disk flux. It is not surprising that such a weak spectral contribution lends itself to detection in the optical only during the deep eclipse when the disk light is vastly diminished.

\subsection{V1309 Ori}

V1309 Ori (RX J0515.6+0105) is listed as an eclipsing magnetic CV with the longest known orbital period for a polar $(7.98 \mathrm{hr})$ and an M0 secondary star (Garnavich et al. (1994). These authors note that a normal M0V is not large enough to fill the Roche lobe in such a long period system. A low resolution infrared spectrum was presented by Harrop-Allin et al. (1997) that revealed H I and He I emission, but no features that they could attribute to the secondary star. They suggested that the IR spectrum was dominated by cyclotron emission from the $\mathrm{B}=33-55 \mathrm{MG}$ white dwarf primary.

Our Keck spectrum is presented in Figure 4 and covers 0.04 in orbital phase. The Brackett $\gamma$ emission line is seen as well as He 2.056 micron emission. The $\mathrm{H}$ emission line is suggested to be from the gas stream and accretion column of material falling toward the white dwarf magnetic pole. We see no evidence for cyclotron humps, but given the apparent strong B field none would be expected in this wavelength region. The rising blue continuum is consistent with an origin in the Rayleigh-Jeans tail of cyclotron emission; a similar

conclusion was reached by Harrop-Allin et al. (1997). However, the rising continuum is also consistent with the spectral energy distribution in this region for $\mathrm{K}$ and early $\mathrm{M}$ stars.

We clearly detect secondary star features as absorption lines due to $\mathrm{Na} \mathrm{I,} \mathrm{Ca} \mathrm{I,} \mathrm{and}$ Mg I. Figure 4 compares our IR spectrum to single star templates covering K0V to M0V with a best fit (based on line ratios) of K6-K8. No CO absorption is detected from the secondary star in V1309 Ori although it should be for this temperature (spectral type) as 
can be seen in the template spectra. For an orbital period of $8 \mathrm{hr}$, the secondary star would be expected to be a G3V ((Smith \& Dhillon 1998) and modeled to have a mass of 1.19 $\mathrm{M}_{\odot}$ and a radius of $0.98 \mathrm{R}_{\odot}$ (Howell et al., 2001). Our $K$-band spectrum seems to clearly

eliminate the possibility that the secondary is such an early G star and we suggest that the donor is indeed near $\mathrm{K} 7$ in spectral type but is a subgiant star as has been shown to exist in the similar orbital period magnetic CV AE Aqr (Harrison et al., 2004a).

V1309 Ori presents a mystery given its lack of $\mathrm{CO}$ absorption. All other polars have shown normal abundance secondary stars with $\mathrm{CO}$ absorption in their $K$-band spectra (Harrison et al. 2005) while a lack of CO has been observed in most dwarf novae. BY Cam does show an enhanced NV/CIV ratio, however, suggestive of CNO processed material accreted from the secondary (Bonnet-Bidaud \& Mouchet, 1987) but no $K$-band spectrum of its donor star has yet been obtained. AE Aqr, the odd intermediate polar, shows an enhanced NV/CIV ratio as well as very weak CO (Harrison et al. 2007). Perhaps V1309 Ori shares some property with AE Aqr or BY Cam leading to its lack of CO absorption. It would be hard to reconcile that V1309 Ori contains a magnetic field strength similar to IPs given the cyclotron spectrum observed and modeled by Garnavich et al. (1994).

\subsection{SS Aur}

SS Aur is a fairly normal dwarf nova with an orbital period of 4.38 hours, therefore above the CV period gap. The binary inclination (38 degrees) and hot white dwarf primary (30,000K: Lake \& Sion 2001) both tend to produce a bright, rising blue continuum. Our $K$-band spectrum (Figure 5) shows the blue continuum rise as well as broad, double-peaked emission lines due to $\operatorname{Br} \gamma$ and He I. Absorption features from the secondary star are also easily observed in Figure 5 and agree with a M4V star, not with the expected secondary type of late K to early M (Smith and Dhillon 1998). We also note that Na I and Ca I are 
a bit too strong for a M4V while the spectrum contains completely normal looking $\mathrm{CO}$ absorption bands (for the observed secondary type). SS Aur is one of the rare DN which have relatively normal $\mathrm{CO}$.

The $K$-band spectrum shown in Figure 5 is consistent with solar abundances in that the line ratios agree with our $\mathrm{M} 4 \mathrm{~V}$ template while the bluer slope herolds the the presence of a hot white dwarf and accretion disk in the SS Aur system. According to AAVSO records our spectrum was obtained in quiescence, 10-12 days prior to a normal outburst. SS Aur contains one of the hottest white dwarfs known in a dwarf nova due to its high mass transfer

rate estimated at $10^{-10} \mathrm{M}_{\odot} \mathrm{yr}^{-1}$ (Lake and Sion 2001). This mass transfer rate is very close to the critical rate for a solar mass white dwarf such that DN outbursts would not occur. This high mass transfer rate may indicate that SS Aur is a relatively new CV, just formed above the period gap (see Howell et al. 2001).

It is interesting to compare SS Aur with U Gem. These two DN have similar orbital periods yet their CO behavior is quite different (c.f., Harrison et al., 2004). SS Aur is a high mass transfer system, thus we would expect an extensive accretion disk. Completely normal C abundance has been noted for this object (Godon et al. 2008) and we see normal looking $\mathrm{CO}$ absorption in the $K$-band spectrum. U Gem, on the other hand, has a lower mass transfer rate, high inclination, and weak $\mathrm{CO}$ absorption. Therefore the difference in observed $\mathrm{CO}$ absorption may be due to the difference in binary inclination or mass transfer rate although the issue is not so clear (see $\S 4$ ).

\section{6. $\quad$ BPM 71213}

This binary consists of a non-magnetic WD plus an M dwarf with an orbital period of $4.33 \mathrm{hr}$. The binary is non-interacting, presumably a pre-CV, and the secondary star shows 
chromospheric activity (Kawka et al., 2002). These same authors determine the secondary star to be M2.5 V.

Our IR spectrum (Figure 6) shows typical absorption features of an M2-3 V star including normal strength Mg I (2.11 \& 2.28 microns) and CO. Tappert et al. (2007) also observed BPM 71213 in the $K$-band and note absorption features apparently weaker than expected for an M2-3 V star. The higher $\mathrm{S} / \mathrm{N}$ in the continuum may be the reason why we find normal absorption depths for all the absorption features, including CO. We observed BPM 71213 on both nights but see no difference in the $K$-band spectrum.

\subsection{QS Vir $=$ EC 13471-1258}

EC 13471-1258 is suggested to be a hibernating CV albeit with an orbital period $\left(\mathrm{P}_{\text {orb }}=3.6 \mathrm{hr}\right.$ ) outside of the usual $\mathrm{CV}$ period gap of $\sim 2$ to $\sim 3 \mathrm{hr}$. If the mass transfer has stopped in this $\mathrm{CV}$, it appears to have stopped quite early with respect to its crossing the 2-3 hour period gap. The binary contains a non-magnetic DA white dwarf and a completely normal, solar abundance, chromospherically active M3.5-4 V secondary star (O'Donoghue et al., 2003). The white dwarf is seen to have a rapid rotation possibly suggestive of evidence for past mass accretion.

Our infrared spectrum (Fig. 6) matches well with a template spectrum of a normal, single M3-4V star, and thus is in good agreement with the O'Donoghue et al., (2003) result. Tappert et al. (2007) also observed this object in the $K$-band and note absorption features weaker than expected for an M4V star. As for BPM 71213, our higher S/N in the continuum may be the reason why we find normal absorption depths for the absorption features, including $\mathrm{CO}$.

The M4V secondary is noted to be very active, flaring often, and showing the typical 
signs of chromospheric activity (O'Donoghue et al., 2003). Our IR spectrum reveals no evidence for this behavior, however that is completely expected as chromospheric activity does not manifest itself in the higher energy hydrogen lines due to their formation deep in the stellar photosphere (see $\S 4.2$, Howell et al., 2006).

The model fit to UV spectroscopy of the white dwarf (O'Donoghue et al., 2003) implies a distance of only 48 pc and would make EC 13471-1258 the second closest CV, after WZ Sge $(\mathrm{d}=43 \mathrm{pc})$, if its CV nature is confirmed. The continuum flux in the $\mathrm{V}$ band is equally

split between the two stars while our $K$-band measurement will contain $<3 \%$ contamination from the white dwarf. Assuming a $100 \%$ contribution from a normal M4V star $\left(\mathrm{M}_{K}=\right.$ 7.4) and convolving a $\mathrm{K}$ filter with our spectrum (giving $\mathrm{K}=11$ ), we find a distance to EC 13471-1258 of 52 pc in close agreement with the previous determination.

\subsection{SDSS0743, SDSS0757, SDSS0830}

These three possible pre-CV non-interacting binaries were discovered from the SDSS spectra taken of red+blue objects (Silvestri et al. 2006a, 2007). An iterative fit to the spectra with white dwarf and secondary star models produced estimates for the temperatures of the white dwarfs and the spectral types of the secondaries (Silvestri 2007). Additional followup spectra and photometry revealed short orbital periods of 3-4 hrs were likely (Silvestri et al. 2006b). We present our Keck $K$-band spectra for all three in Figure 7.

SDSS0743 is a $g=17.34$ system with an M4V star determined from the optical spectrum. Our $K$-band spectrum looks slightly earlier then M4V although the Na I absorption is too strong for this type given the depth of the CO absorption. The Na I strength would argue for a slightly later star, M5-M6V.

SDSS0757 is a $g=16.36$ binary with an M2V secondary as determined from optical 
spectroscopy. The $K$-band spectrum shown in Figure 7 may show weak $\operatorname{Br} \gamma$ emission but confirmation is required. The weakness in the $K$-band of the MgI/AlI complex $(2.11$ microns) and the relative strength of CaI absorption at 2.26 microns argues for a slightly later spectral type near M3-M4V.

SDSS0830 has a $g$ magnitude of 18.50 and an M0V secondary as determined from optical spectra. Our Keck spectra (Fig 7) is of low S/N but generally consistent with this type or slightly later (M1-M2V) and appears to contain blue continuum contamination from the white dwarf.

\subsection{SDSS0837}

This faint $(g=19)$ LARP (Low Accretion Rate Polar) was identified from its unusual SDSS spectrum, which showed cyclotron humps near $4500 \AA$ and $6200 \AA$ and $\mathrm{TiO}$ bands consistent with an M5V secondary (Schmidt et al. 2005). Their followup spectropolarimetry confirmed high polarization and determined that the cyclotron humps were harmonics 4 and 3 in a field of 65 MG. However, a polarization of the opposite sign was also apparent between the harmonics, indicating that a second accretion pole with a different field strength was also present. CCD photometry indicated an orbital period of either 3.18 or $3.65 \mathrm{hr}$. Later XMM data and optical data (Hilton et al. 2009) confirmed a very low accretion rate, consistent with no accretion shock at the poles and determined the correct orbital period to be $3.18 \mathrm{hr}$. While it is clear that LARPs do not have mass transfer through the usual ballistic stream, it is likely they are still accreting via a wind from the secondary that is channelled to the magnetic poles (Howell 2008). Due to the very low accretion rates and especially to the low temperatures of the white dwarfs in LARPS (under 10,000K), it has been suggested that Roche-lobe overflow has not taken place and these objects may be pre-Polars (Schmidt et al. 2005; Schwope et al. 2009). 
Our infrared spectrum is shown in Figure 8. While of low $\mathrm{S} / \mathrm{N}$, we note the presence of weak Na I and CO absorption. Ca I may be present, but the continuum noise level for this relatively faint target prevents us from a positive identification. The secondary star in this LARP looks like a normal M4-5V star, similar to that observed in SDSS0743.

\subsection{ST LMi}

The magnetic CV ST LMi has been the subject of many studies, most performed with optical or higher energy observations and aimed at an understanding of the magnetic white dwarf or the accretion geometry and processes (e.g., Cropper and Horne, 1994; Stockman and Schmidt 1996, Robertson et al., 2008). The white dwarf has a weak magnetic field for a polar, 11.5 MG, and the binary orbital period is short at 1.9 hours, below the CV period gap.

Figure 9 shows four consecutive spectral sets we obtained at Keck covering $\sim 30$ min or about 0.25 of an orbit. During this time, it is apparent that the strengths of the absorption features, assumed to arise from the secondary star, vary, getting stronger toward the end of the spectral sequence (bottom of Figure 9). Campbell et al. (2008) show that the $K$-band magnitude of ST LMi changes by 0.8 over an orbit causing absorption features to weaken as the $\mathrm{n}=4$ cyclotron harmonic dominates the continuum. During times of no, to weak cyclotron contamination, the secondary star is observed to be M6V. Emission lines of $\mathrm{H}$ and $\mathrm{He}$ are also apparent showing equivalent width variations and profile shape changes, both typical for this star as illustrated in Robertson et al. (2008).

Changes in line strength, particularly Na I absorption, were noted previously for ST LMi by Howell et al (2000) and attributed to star spots on the surface of the secondary star. We also see (as did Howell et al. 2000) that CO absorption bands are present, expected 
for a polar (Harrison et al. 2005) but they change strength with time. Changing spectral line profiles are common for polars which present the observer with a changing view of the cyclotron emission region during its orbit.

\subsection{RZ Leo}

This little studied, faint dwarf nova has an orbital period of $1.84 \mathrm{hr}$ (Mennickent and Tappert 2001). Previous work has been nearly exclusively photometric and as such, we know little about the component stars in RZ Leo. Mennickent \& Tappert (2001) present a RV solution with a K1 amplitude of $\sim 80 \mathrm{~km} / \mathrm{sec}$.

Our IR spectrum presented in Figure 10 covers a total time period of 1 hour, or about one-half of the orbital period. The emission lines due to Brackett $\gamma$ (2.16 microns) and He 2.056 microns are of moderate strength and appear double-peaked. They are formed in the accretion disk and suggest a high inclination, although no eclipses are observed.

Absorption features from the secondary star are weakly detected and consist of Na I, (strong) $\mathrm{Ca}$ I, and CO molecular absorption edges at 2.29, 2.32, and 2.35 microns. The third $\mathrm{CO}$ absorption band is blended with the Na I doublet at 2.35 microns, and the fourth available $\mathrm{CO}$ absorption (2.38 microns) falls in a noisy continuum region and we cannot be sure of its detection. While the absorption features are filled in by accretion flux, their relative depths are useful as spectral type indicators. We find the secondary star in RZ Leo to be consistent with an M3-M4 V star, consistent with expectations (Smith \& Dhillon 1998). RZ Leo is only the third dwarf nova (after IP Peg and SS Aur) to show normal CO absorption in its infrared spectrum. WZ Sge shows CO as well, but in emission (Howell et al., 2004). 


\subsection{GW Lib}

This short period CV has relatively rare superoutbursts and belongs to the Tremendous Outburst Amplitude Dwarf nova (TOAD) class of CV (Howell, Szkody, \& Cannizzo 1995),

similar to WZ Sge. Our summed IR spectrum covers nearly one complete orbital cycle and is presented in Figure 10. We see a strong, narrow emission line $(\mathrm{Br} \gamma)$ from the accretion disk. The single-peaked nature suggests that the binary has a low inclination as has been determined previously ( $i=11$ degrees, Szkody et al., 2002; Thorstensen et al. 2002). A typical double-peaked accretion disk emission line profile in a system such as GW Lib (e.g., using WZ Sge as an example, Skidmore et al., 2000) would have a velocity width (FWZI) near $1200 \mathrm{~km} / \mathrm{sec}$ for an inclination of 90 degrees. At 11 degrees, this velocity width would be expected to be near $200 \mathrm{~km} / \mathrm{sec}$, essentially at our spectral resolution but in agreement with the single-peaked line we see.

Our $K$-band spectrum has moderate $\mathrm{S} / \mathrm{N}$ in the continuum but no explicit absorption features from the secondary star are detected. However it does seem that the CO band $(+$ water vapor) continuum break, present in M8-9 stars and L type brown dwarfs (see Kendall et al. 2004), may be marginally detected starting at 2.29 microns and extending redward. Given the strength of the white dwarf continuum (Szkody et al. have shown that the UV and optical continua are dominated by the WD) and some accretion disk contribution (based on the presence of emission lines and the low inclination) we are not surprised at the lack of secondary star absorption features. If we are detecting the CO continuum break, our best fit donor star would be of spectral type later then M9 V having a mass of less than $0.06 \mathrm{M} \odot$.

Szkody et al., (2002) determined a mass of $0.8 \mathrm{M} \odot$ for the pulsating white dwarf in GW Lib from fitting the HST spectrum with a dual temperature white dwarf model. Taking K1=40 km/sec, as found by Szkody et al. and Thorstensen et al. (2002) from 
optical spectroscopy and our mass limit above, $q \leq 0.075$ and the K2 velocity amplitude would be near $500 \mathrm{~km} / \mathrm{sec}$.

\section{Discussion}

Our sample of pre- and present day CVs, both with magnetic and non-magnetic white dwarfs, was obtained in order to provide a more coherent picture of the presence or absence of CO absorption in the IR spectra of these stars. Harrison et al. (2004,2005) set the stage by showing the apparent presence of normal CO absorption in polars and the apparent lack of or weak $\mathrm{CO}$ absorption (but the presence of $\mathrm{O}$ ) in dwarf novae. Taking this information, it has been suggested that the secondary stars in dwarf novae are highly evolved CNO burners, while those in polars are younger main sequence stars of near solar abundance. This scenario requires a different evolution for pre-CVs which become dwarf novae as compared to pre-CVs that become polars - the latter group being essentially unknown (Liebert et al. 2005). The results presented here appear to add further confusion to the issue of the evolutionary path of CVs. We find that the polar V1309 Ori has extremely weak CO features, in contrast to the result for all other polars (Harrison et al. 2005), while the secondary in the mainstream non-magnetic CV, SS Aur, appears to have normal CO features. Both of these objects have a few characteristics that set them apart from other members of their respective classes, but they certainly do not show any behavior that is not seen in other members of their classes. We confirm the suggestion by Dhillon et al. (2002) that there is no evidence for enhanced $13 \mathrm{CO}$ absorption in V471 Tau. Thus, none of the so-called pre-CVs presented here, or elsewhere, show any sign of abundance peculiarities.

The objects observed in this study are listed in Table 2 and our former $K$-band studies

in the literature are listed in Table 3. Taken together, these observations seem to cloud the issue of $\mathrm{CO}$ absorption as we find that all the pre-CVs, which all have non-magnetic white 
dwarfs and are thought to evolve into dwarf novae, show apparent normal abundances including $\mathrm{CO}$ absorption. We also find that the supposedly hibernating CV, QS Vir, shows normal CO absorption as well. In addition, one polar (V1309 Ori), does not show CO absorption but has the somewhat non-typical polar properties of a very long orbital period and a subgiant secondary. There is no single theory to reconcile these results that doesn't strain credulity. If magnetic and non-magnetic CV evolution is identical, then we must ignore the growing evidence from UV and IR observations that there appear to be abundance issues in non-magnetic CV systems. This requires us to find a method to preferentially obscure the CO features in the secondary star of disk-accreting systems (CO emission from the accretion disk?), and (simultaneously) excite nitrogen emission over that of carbon. Or we must come up with a method that strongly enhances N, and depletes C, between the pre-CV phase and contact, that only occurs in disk-accreting CVs. This also relegates some subset of CVs, including U Gem, WZ Sge, VY Aqr, V1309 Ori, AE Aqr, GK Per, CH UMa, and perhaps even SS Cyg (Robinson \& Bitner 2009) and RU Peg, to the "oddball" bin.

The idea of actual low $\mathrm{C}$ abundance would likely mean that some of the secondary stars follow a different evolution to become a CV with a seemingly high dependence on their white dwarf's magnetic properties. Another possibility is that some of the present day CVs we observe are simply systems which contain older secondary stars, stars that are highly evolved nearing or just past their main sequence lifetime. If this latter idea is true, then it implies that essentially all polars are younger systems and any containing evolved secondaries are essentially unknown or observationally selected against. Additionally, the present sample of pre-CVs seems not to contain any evolved stars soon to be CV donors. We could try to accept that pre-CVs secondaries go through an extremely rapid evolution including $\mathrm{CNO}$ burning between the present epoch and their becoming a dwarf novae or that all the present day pre-CVs will not actually end up as dwarf novae. But both of 
these scenarios seem highly ad hoc. It may be that the entire binary undergoes a different evolutionary path depending on the formation of a magnetic or non-magnetic WD (Tout et al., 2008) with some non-magnetic systems taking longer until contact allowing some donors to evolve.

Another scenario for low $\mathrm{C}$ abundance may be that the secondary star collects CNO processed material from the white dwarf during DN outbursts and this material covers and hides the normal abundance photosphere providing us with a view showing an apparent lack of CO. This idea has many flaws, not the least of which are the amazingly small solid angle the secondary subtends in which to receive most of the material, the problem that this material would have to spread to cover the entire secondary star surface, and the fact that a convective atmosphere will mix the material into the star's interior very fast, a few tens of minutes (Kawaler 2008, priv. comm).

Exploring another option, Howell et al. (2004) noted that WZ Sge, a non-magnetic $\mathrm{CV}$, shows $\mathrm{CO}$ emission in its $K$-band spectrum. The most likely formation site for the CO emission was in the outer cool dense regions of the accretion disk. These regions are dense and cool enough to shield the CO molecules from the UV emission able to disassociate them. Furthermore, Howell et al. (2008) found that the outer accretion disk regions in WZ Sge were indeed very cool, cool enough for dust to form. The common property linking many of the CVs which show no or weak CO absorption is the fact that they have accretion disks - most of those without accretions disks show normal CO absorption. QS Vir may be the most interesting. It is supposed to be a hibernating non-magnetic CV, thus it was a DN with an accretion disk and will be again, yet it has a normal looking secondary star including $\mathrm{CO}$ absorption.

Can CO emission from the accretion disk fill in the secondary star CO absorption and account for the weak or missing lines? To see if this might work, we choose to model 
the dwarf nova $\mathrm{U}$ Gem which is noted to have very weak to no CO absorption present in its $K$-band spectrum (Harrison et al., 2005b). We produced an artificial CO emission spectrum, and using the fact that the Br $\gamma$ disk emission line in U Gem has a FWHM velocity of $\sim 1750 \mathrm{~km} / \mathrm{s}$, we broadened our model CO emission by $1500 \mathrm{~km} / \mathrm{s}$ so as to approximate a more "distant" emission region (i.e., the outer disk). We next took an M4V template spectrum (the spectral type of the secondary star in U Gem) and summed it with our CO emission model. The M4V template spectrum we used is shown at the bottom of the plot. It has a velocity broadening of $90 \mathrm{~km} / \mathrm{s}$ applied to get it to look more or less like the observed secondary star features present in the U Gem spectrum.

We normalized the first overtone of the CO emission spectrum to exactly match the absolute depth of the $\mathrm{CO}$ overtone absorption band in the template spectrum to make a best attempt to "fill-in" the CO absorption spectrum. We then replaced the red end (redward of 2.28 microns) of the U Gem $K$-band spectrum (orbital phase 0.20) with our summed model scaled to match the observed continuum at 2.28 microns (Figure 11). While we did not produce a full disk model spectrum with added CO emission, this exercise was intended to illustrate whether CO emission could fill-in or hide the expected M star molecular absorption. The spectrum labeled "100\%" in Fig. 11 is our initial model as just described while the one labeled " $50 \%$ " is the same process but with the CO overtone emission at only $50 \%$ of its initial strength. We note that in the $100 \%$ case, the narrow CO absorption features create troughs at the emission line peaks (similar to the HI lines in GK Per and SS Cyg as shown in Fig. 10 of Harrison et al., 2009). Even in the "50\%" spectrum is choppy with emission humps on both sides of each of the narrower $\mathrm{CO}$ absorption features. However, when the $\mathrm{S} / \mathrm{N}$ of the red continuum is poor, this choppiness may not be noticeable as in, for example, the spectrum presented here for RZ Leo and other fainter DN with $K$-band spectra in the literature. 
If $\mathrm{CO}$ emission from the accretion disk is the cause of the weak or absent secondary star CO absorption then why do not the systems where the $K$-band spectra are completely dominated by disk emission show CO emission? Only WZ Sge has shown clear CO emission and we noted earlier that this same star is also the only CV which is carbon rich. The CV systems where the disk flux dominates the $K$-band (i.e., no secondary star seen) are ones with high mass transfer rates and hot accretion disks, perhaps too hot to contain cool regions able to form $\mathrm{CO}$. Or they are low inclination systems where the hot inner disk and white dwarf continuum light outshine the remaining disk (including any $\mathrm{CO}$ emission present).

There are some non-magnetic CVs which show weak or normal CO absorption strengths yet also have accretion disks. We could try to make arguments such as in RZ Leo we see only weak $\mathrm{CO}$ absorption perhaps related to its high inclination ( $i=65$ degrees) or its low mass transfer rate. SS Aur, on the other hand, has a moderate inclination ( $i=38$ degrees) but contains a hot $(30,000 \mathrm{~K}) \mathrm{WD}$. Perhaps this hot star provides sufficient UV flux so as to not allow $\mathrm{CO}$ to form in the disk. Maybe the carbon abundance is just not high enough to produce $\mathrm{CO}$ emission in the accretion disk? Do accretion disks know how to make just enough CO emission to fill-in the secondary stars CO absorption in the "no" cases and just too little in the weak cases? Why is WZ Sge so far the only disk system to show CO emission? Does the lack of CO absorption in a DN remain constant over the orbit and over time? All good questions without good answers.

Based on weak CO absorption, parallaxes, and other abundance anomalies, Harrison et al. (2004a) have argued that the CVs SS Cyg, RU Peg AE Aqr, and GK Per contain subgiant secondary stars. We show in this paper that the long-period polar V1309 Ori is likely to contain a subgiant secondary as well. Binary evolution models of CVs (e.g., Howell et al., 2001) show that systems in the range of about 3 to 5 hours have bloated 
secondary stars, that is their secondaries are too large for their mass compared to main sequence models. This fact may make some secondaries appear to be subgiants but cannot alone explain the weakness of $\mathrm{CO}$ absorption, $13 \mathrm{CO}$, or other anomalies in the spectra. Beuermann et al. (1998) explored evolutionary scenarios in which the secondary stars had undergone nuclear evolution prior to mass transfer leading to the suggestion that many long period CVs should have evolved donor stars. Barraffe and Kolb (2000) have shown that some observational properties of CVs above the period gap can be explained if evolutionary models include secondary stars which have some $\mathrm{H}$ depletion in their core, including secondaries which are substantially evolved off the main sequence (i.e., subgiants). Detailed abundance studies of CV secondaries, while a difficult observational task, is needed to place any of these arguments on a firm foundation.

The reality is that we do not know the exact mechanism by which CO emission could be produced in the accretion disks or in what fraction of disks it might be produced. Some CVs show evidence in both the UV (high $\mathrm{N} / \mathrm{C}$ ratios) and the $K$-band (weak or absent CO) to suggest that the secondary stars are highly evolved. Our sample of 24 disk systems discussed here does not provide any consistent clues to settle the discussion. Perhaps a variety of mechanisms are operating simultaneously within the binary systems. To date, there does not seem to be a single answer which can account for all the observations regarding the weak or absent $\mathrm{CO}$ absorption in the secondary stars of cataclysmic variables.

We wish to thank the Keck II OAs Heather, Chuck, Terry, and Jason for their help with our observations and Jim Lyke for his help in the instrument setup. SH thanks the ESO DFGR for Financial support and Marina Orio and the University of Padova Observatory, for their hospitality and the use of a 16th century Specola tower office. We thank the anonymous referee for their detailed and careful reading of the manuscript.

Data presented were obtained at the W. M. Keck Observatory, which is operated as 
a scientific partnership among the California Institute of Technology, the University of California, and the National Aeronautics and Space Administration. The Observatory was made possible by the generous financial support of the W. M. Keck Foundation. The authors wish to recognize and acknowledge the very significant cultural role and reverence that the summit of Mauna Kea has always had within the indigenous Hawaiian community. We are most fortunate to have the opportunity to conduct observations from this mountain. 
Table 1. Observing Log

\begin{tabular}{|c|c|c|c|}
\hline Star & UT Date & UT Start & Total Exp. Time \\
\hline V1309 Ori & 4 Mar 2007 & $5: 32$ & $16 \min$ \\
\hline V471 Tau & 4 Mar 2007 & $5: 22$ & $12 \mathrm{sec}$ \\
\hline QS Vir & 4 Mar 2007 & $14: 48$ & $4 \mathrm{~min}$ \\
\hline BPM 71213 & 4 Mar 2007 & $5: 09$ & $4 \mathrm{~min}$ \\
\hline BT Mon & 4 Mar 2007 & $6: 22$ & $40 \mathrm{~min}$ \\
\hline GW Lib & 4 Mar 2007 & $15: 23$ & $64 \mathrm{~min}$ \\
\hline SS Aur & 5 Mar 2007 & 7:09 & $32 \min$ \\
\hline RZ Leo & 5 Mar 2007 & $11: 58$ & $16 \mathrm{~min}$ \\
\hline ST LMi & 4 Mar 2007 & $13: 11$ & $64 \min$ \\
\hline SDSS0837 & 4 Mar 2007 & $10: 43$ & $8 \min$ \\
\hline SDSS0830 & 4 Mar 2007 & $9: 44$ & $8 \mathrm{~min}$ \\
\hline SDSS0757 & 4 Mar 2007 & $8: 49$ & $8 \mathrm{~min}$ \\
\hline SDSS0743 & 4 MAr 2007 & $7: 56$ & $16 \min$ \\
\hline HS1136 & 4 Mar 2007 & $12 ; 30$ & $4 \mathrm{~min}$ \\
\hline
\end{tabular}


Table 2. Status of CO absorption-This Sample

\begin{tabular}{cccccc}
\hline \hline \multirow{2}{*}{ Star } & Type & $\mathrm{P}_{\text {orb }}(\mathrm{hr})$ & Magnetic? & CO Ab. ${ }^{a}$ ? & Notes \\
\hline HS1136 & pre-CV & 20.1 & $\mathrm{~N}$ & $\mathrm{~N}$ & WD dominates IR flux \\
V471 Tau & pre-CV & 12.5 & $\mathrm{~N}$ & $\mathrm{Y}$ & \\
BT Mon & DN & 7.99 & $\mathrm{~N}$ & $\mathrm{~N}$ & Ecl., disk dominates IR flux \\
V1309 Ori & polar & 7.98 & $\mathrm{Y}$ & $\mathrm{N}$ & Ecl. \\
SDSS0743 & pre-CV & 4.6 & $\mathrm{~N}$ & $\mathrm{Y}$ & \\
SS Aur & DN & 4.38 & $\mathrm{~N}$ & $\mathrm{Y}$ & \\
BPM 71213 & pre-CV & 4.33 & $\mathrm{~N}$ & $\mathrm{Y}$ & \\
QS Vir & hibernating CV & 3.6 & $\mathrm{~N}$ & $\mathrm{Y}$ & =EC 13471 \\
SDSS0757 & pre-CV & 3.5 & $\mathrm{~N}$ & $\mathrm{Y}$ & \\
SDSS0837 & LARP & 3.18 & $\mathrm{Y}$ & $\mathrm{Y}$ & pre-polar? \\
SDSS0830 & pre-CV & 2.9 & $\mathrm{~N}$ & $\mathrm{Y}$ & \\
ST LMi & polar & 1.91 & $\mathrm{Y}$ & $\mathrm{Y}$ & cyclotron cont. \\
RZ Leo & DN & 1.84 & $\mathrm{~N}$ & $\mathrm{~W}$ & \\
GW Lib & DN/TOAD & 1.33 & $\mathrm{~N}$ & $\mathrm{~N} ?$ & \\
\hline \hline
\end{tabular}

${ }^{a} Y=$ appears normal for spectral type; $\mathrm{W}=$ appears weaker than normal for spectral type; $\mathrm{N}=$ not present; $\mathrm{N}$ ?=maybe present, too low $\mathrm{S} / \mathrm{N}$ to be certain. 
Table 3. Status of CO Absorption-Previous Sample ${ }^{a}$

\begin{tabular}{|c|c|c|c|c|c|}
\hline Star & Type & $\mathrm{P}_{\text {orb }}(\mathrm{hr})$ & Magnetic? & $\mathrm{CO} \mathrm{Ab}^{b} ?$ & $\operatorname{Ref}^{c}$ \\
\hline AE Aqr & DQ & 9.86 & $\mathrm{Y}$ & $\mathrm{W}$ & 7 \\
\hline SY Cnc & DN & 9.12 & $\mathrm{~N}$ & $\mathrm{~N}$ & 5 \\
\hline RU Peg & $\mathrm{DN}$ & 8.99 & $\mathrm{~N}$ & $\mathrm{~W}$ & 5 \\
\hline $\mathrm{CH} \mathrm{UMa}$ & $\mathrm{DN}$ & 8.23 & $\mathrm{~N}$ & $\mathrm{~W}$ & 5 \\
\hline MU Cen & DN & 8.21 & $\mathrm{~N}$ & $\mathrm{~W}$ & 5 \\
\hline $\mathrm{AC} \mathrm{Cnc}$ & NL & 7.21 & $\mathrm{~N}$ & $\mathrm{~W}$ & 5 \\
\hline EM Cyg & DN & 6.98 & $\mathrm{~N}$ & $\mathrm{Y}$ & 5 \\
\hline V426 Oph & $\mathrm{DN}$ & 6.85 & $\mathrm{~N}$ & $\mathrm{Y}$ & 5 \\
\hline SS Cyg & $\mathrm{DN}$ & 6.60 & $\mathrm{~N}$ & $\mathrm{Y}$ & 5 \\
\hline AH Her & Z Cam & 6.20 & $\mathrm{~N}$ & $\mathrm{~W}$ & 5 \\
\hline BV Pup & $\mathrm{DN}$ & 6.35 & $\mathrm{~N}$ & $\mathrm{~W}$ & 5 \\
\hline EX Dra & $\mathrm{DN}$ & 5.04 & $\mathrm{~N}$ & Y & 4 \\
\hline SDSS1553+55 & polar & 4.39 & $\mathrm{Y}$ & $\mathrm{Y}$ & 3 \\
\hline TW Vir & $\mathrm{DN}$ & 4.38 & $\mathrm{~N}$ & $\mathrm{~N}$ & 4 \\
\hline SS Aur & $\mathrm{DN}$ & 4.29 & $\mathrm{~N}$ & $\mathrm{~W}$ & 4 \\
\hline U Gem & DN & 4.25 & $\mathrm{~N}$ & W & 4 \\
\hline UU Aql & $\mathrm{DN}$ & 3.92 & $\mathrm{~N}$ & $\mathrm{~N}$ & 4 \\
\hline IP Peg & DN & 3.80 & $\mathrm{~N}$ & $\mathrm{Y}$ & 4 \\
\hline RR Pic & $\mathrm{CN}$ & 3.48 & $\mathrm{~N}$ & $\mathrm{~W}$ & 4 \\
\hline AM Her & polar & 3.09 & $\mathrm{Y}$ & $\mathrm{Y}$ & 4 \\
\hline AR UMa & polar & 1.93 & $\mathrm{Y}$ & $\mathrm{Y}$ & 3 \\
\hline ST LMi & polar & 1.90 & $\mathrm{Y}$ & $\mathrm{Y}$ & 3 \\
\hline MR Ser & polar & 1.89 & $\mathrm{Y}$ & $\mathrm{Y}$ & 4 \\
\hline VV Pup & polar & 1.67 & $\mathrm{Y}$ & $\mathrm{Y}$ & 2,3 \\
\hline VY Aqr & TOAD & 1.51 & $\mathrm{~N}$ & $\mathrm{~N}$ & 1 \\
\hline EI Psc & DN? & 1.07 & $\mathrm{~N}$ & $\mathrm{~N}$ & 1 \\
\hline WZ Sge & TOAD & 1.35 & $\mathrm{~N}$ & $\mathrm{E}$ & 6 \\
\hline
\end{tabular}

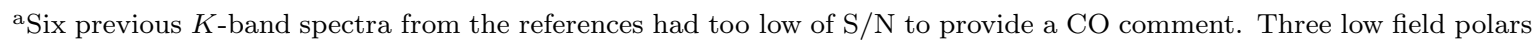
were not included as they have their $K$-band continuum dominated by cyclotron emission. AC Cnc and EX Dra eclipse.

${ }^{\mathrm{b}} \mathrm{Y}=$ appears normal for spectral type; $\mathrm{W}=$ appears weaker than normal for spectral type; $\mathrm{N}=$ not present; $\mathrm{E}=\mathrm{emission}$.

${ }^{\mathrm{c}} 1=$ Harrison et al., 2009, 2=Howell et al. 2006a, 3=Harrison et al., 2005a, 4=Harrison et al., 2005b, 5=Harrison et al., 2004., 6=Howell et al., 2004, 7=Harrison et al., 2007 


\section{REFERENCES}

Barraffe, I. \& Kolb U., 2000, MNRAS, 318, 354

Beuermann K., Baraffe I., Kolb U., Weichhold M., 1998, A\&A, 339, 518

Bonnet-Bidaud, J. M., \& Mouchet, M. 1987, A\&A, 188, 89

de Bruijne, J. H., Hoogerwerf, R., \& de Zeeuw, P. 2001, A\&A, 367,

Campbell, R., et al., 2008, ApJ, 683, 409

Catalan, M. S., et al., 2001, ASPC, 229, 251

Cropper, M., Horne, K., 1994, MNRAS, 267, 481

Dhillon, V. S., et al., 2002, A\&A, 393, 611

Dhillon, V. S., \& Marsh T. R., 1995, MNRAS, 275, 89

Eggleton, P., 2006, in "Evolutionary Processes in Binary and Multiple Stars", Cambridge University Press Cambridge University Press, UK.

Gansicke, B., et al., 2003, ApJ, 594, 443

Garnavich, P., et al., 1994, ApJ, 435, L144

Godon, P. et al., 2008, ApJ, 679, 1447

Harrop-Allin, M. K., Cropper, M., Potter, S. B., Dhillon, V. S., Howell, S. B., 1997, MNRAS, 288, 1033

Harrison, T. E., Howell, S. B., Szkody, P., et al., 2004, ApJ, 614, 947

Harrison, T. E., Campbell, R., Howell, S. B., Johnson, J. J., 2004a, BAAS, 205, 1915

Harrison, T. E., Osborne, H., \& Howell, S. B., 2004, AJ, 127, 3493

Harrison, T. E., Osborne, H., \& Howell, S. B., 2005b, AJ, 129, 2400

Harrison, T. E., Howell, S. B., Szkody, P., \& Cordova, F., 2005a, ApJ, 632, L123 
Harrison, T. E., et al., 2007, ApJ, 656, 444

Harrison, T. E., Bornak, J., Howell, S. B., Mason, E., Szkody, P., \& McGurk, R., 2009, AJ, 137,4061

Hilton, E., et al., 2009, AJ, 137, 3606

Howell, S. B., Szkody, P., \& Cannizzo, J. K., 1995, ApJ, 439, 337

Howell, S. B., Ciardi, D. R., Dhillon, V. S., Skidmore, W., 2000, ApJ, 530, 904

Howell, S. B., Nelson, L., \& Rappaport, S., 2001, ApJ, 550, 897

Howell, S. B., Harrison, T. E., Szkody, P., 2004, ApJ, 602, L49

Howell, S. B., et al., 2006, ApJ, 652, 709

Howell, S. B., et al., 2006a, AJ, 131,2216

Howell, S. B., et al., 2008, ApJ, 685, 418

Howell, S. B., et al., 2008a, AJ, 136, 2541

Howell, S. B., 2008, in "Short-Period Binary Stars", eds. E. Milone, D. Leahy, and D. Hobill, p. 147, Springer

Ibanoglu, C., Keskin, V.; Akan, M. C.; Evren, S.; Tunca, Z., 1994, A\&A, 281, 811

Kendall, T. R., et al., 2004, A\&A, 416, L17

Lake, J., \& Sion, E. M., 2001, AJ, 122, 1632

Liebert, J., Williams, K. A., Holberg, J. B., Sing, D. K., 2006, PASP, 118, 1528

Liebert, J., et al., 2005, AJ, 129, 2376

Marks, P. B., and Sarna, M. J., 1998, MNRAS, 301, 699

Mennickent, R. E., \& Tappert, C., 2001. A\&A, 372, 563

O'Brien, M. S., Bond, H., \& Sion, E., 2001, ApJ, 563, 971 
O’Donoghue, D., et al., 2003, MNRAS, 345, 506

Rayner, J. T., Cushing, M. C., \& Vacca, W.D., 2009, ApJS, 185, 289

Robertson, J. W., Howell, S. B., Honeycutt, R. K., Kafka, S., Campbell, T., 2008, AJ, 136, 1857

Robsinson, E. L., and Bitner, M. A., 2009, "Wild Stars II", http://www.noao.edu/meetings/wildstars2/wildstars-presentations.php

Rottler, L., Batalha, C.; Young, A.; Vogt, S., 2002, A\&A, 392, 535

Schmidt, G. D. et al. 2005, ApJ, 630, 1037

Smith, D. A., Dhillon, V. S., \& Marsh, T. R., 1998, MNRAS, 296, 465

Smith, D. A., \& Dhillon, V. S., 1998, MNRAS, 301, 767

Silvestri, N. M. S. et al. 2006a, AJ, 131, 1674

Silvestri, N. M. S. et al. 2006b, BAAS, 38, 1128

Silvestri, N. M. S. et al. 2007, AJ, 134, 741

Sing, D., et al., 2004, AJ, 127, 2936

Sing, D., 2009, priv. comm.

Skidmore, W., et al., 2000, MNRAS, 318, 429

Schwope, A.D., Gomez-Moran, A. N/, Schreiver, M. R. \& Gansicke, B. T. 2009, A\&A, 500, 867

Smith, D. A., \& Dhillon, V. S., 1998, MNRAS, 301, 767

Stockman, H. S., Schmidt, Gary D., 1996, ApJ, 468, 883

Szkody, P., Gansicke, B. T., Howell, S. B., Sion, E. M., 2002, ApJ, 575, 796

Tappert, C., et al., 2007, A\&A, 475, 575 
Thorstensen, J. R., Patterson, J., Kemp, J., Vennes, S., 2002, PASP, 114, 1108

Tout, C., et al., 2008, MNRAS, 387, 897

White, J. C., Schlegel, E., \& Honeycutt, K., 1996, ApJ, 456, 777

Warner, B., 1995, in "Cataclysmic Variables", Cambridge University Press 


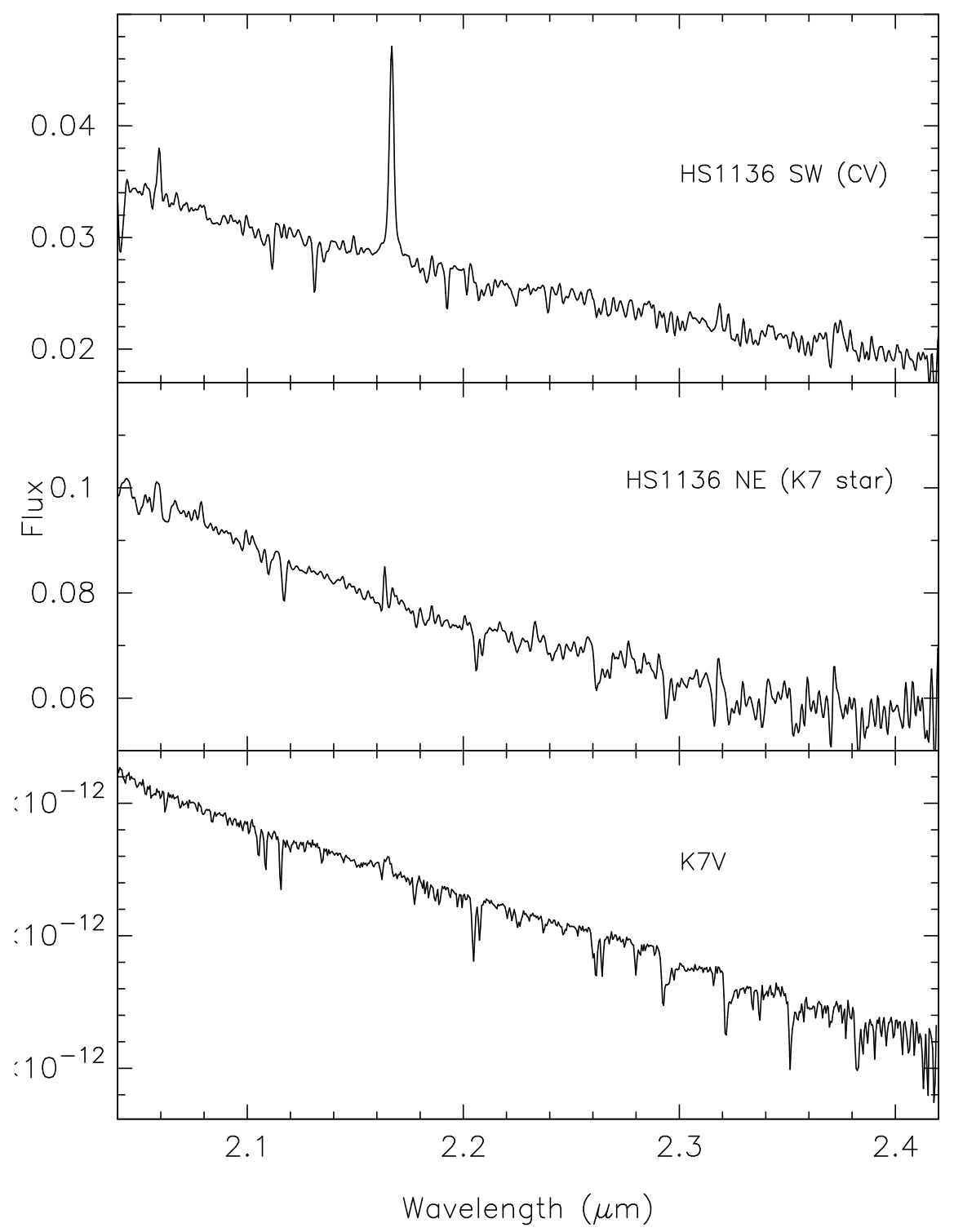

Fig. 1.- Our infrared $K$-band spectrum of the pre-CV HS1136 (top) and its spatially nearby, common proper motion companion K7V star (middle). The bottom panel shows a template single K7V star for comparison. In all the Figures, the Y-axis is relative flux either presented as normalized for our program stars and some templates (set to 1.0 at 2.19 microns) or presented in units of $\operatorname{ergs} / \mathrm{cm}^{2} / \mathrm{sec} / \AA$ for most of the template standards. 


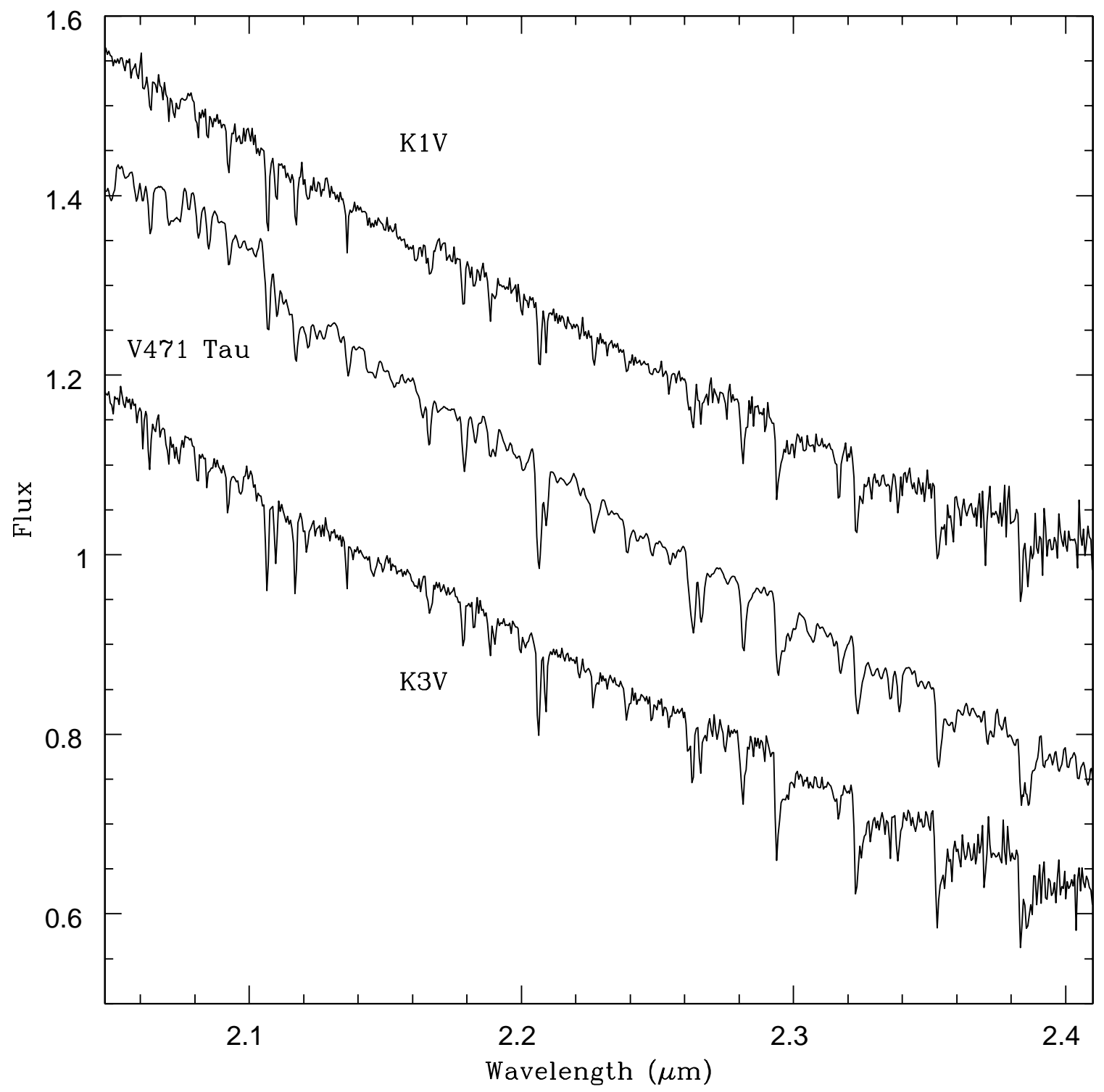

Fig. 2.- Infrared $K$-band spectrum of the canonical pre-CV V471 Tau compared with single star templates for K1V and K3V stars. 


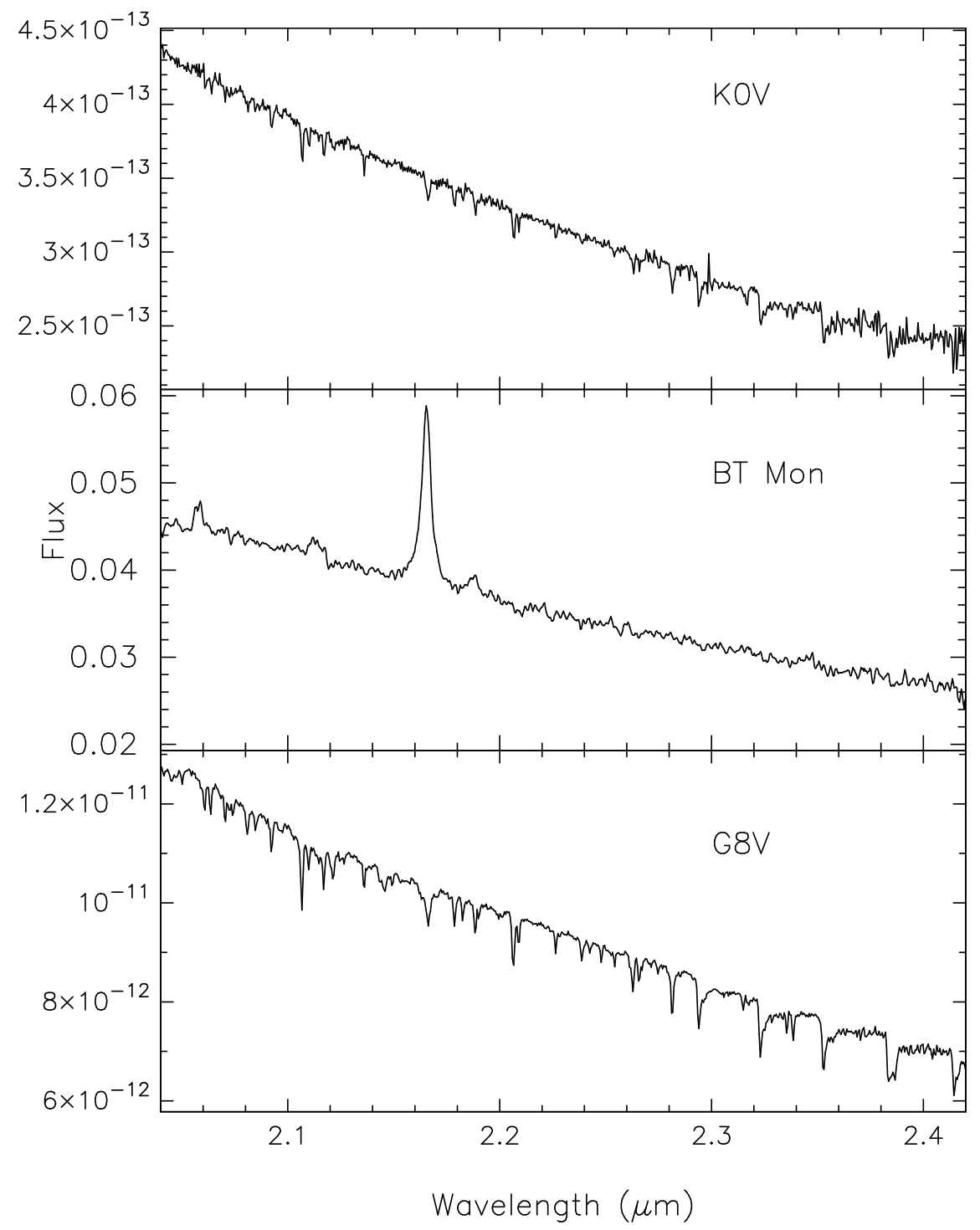

Fig. 3.- Infrared $K$-band spectrum of the old nova BT Mon (containing a G8V secondary star). We show single star templates for K0V and G8V in the figure. 


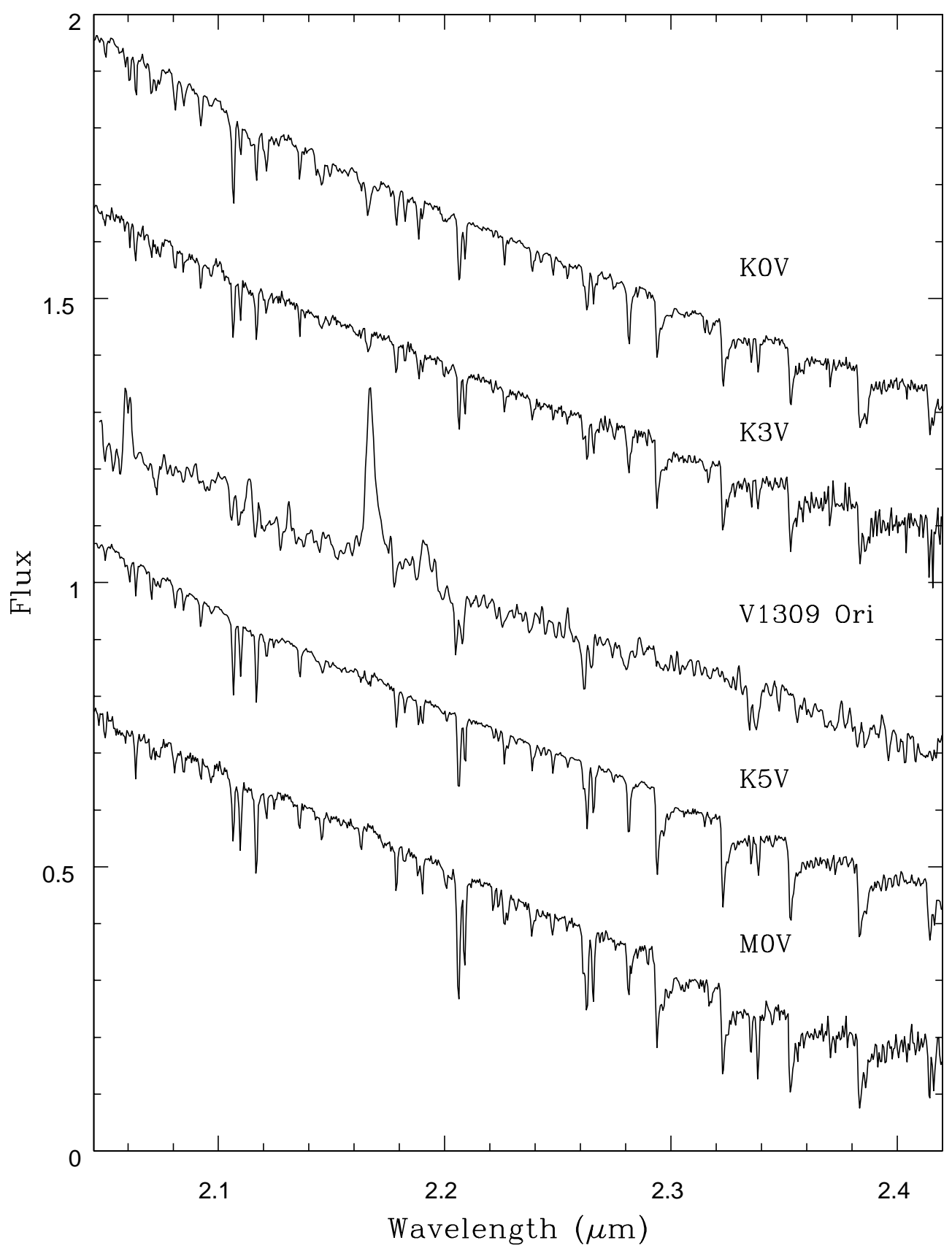

Fig. 4.- Comparison of V1309 Ori $K$-band spectrum with single star template spectra covering M0V to K0V. The line ratios for $\mathrm{NaI}$ to $\mathrm{CaI}$ are consistent with a secondary star of spectral type near K7. Note the complete absence of CO absorption in V1309 Ori. 


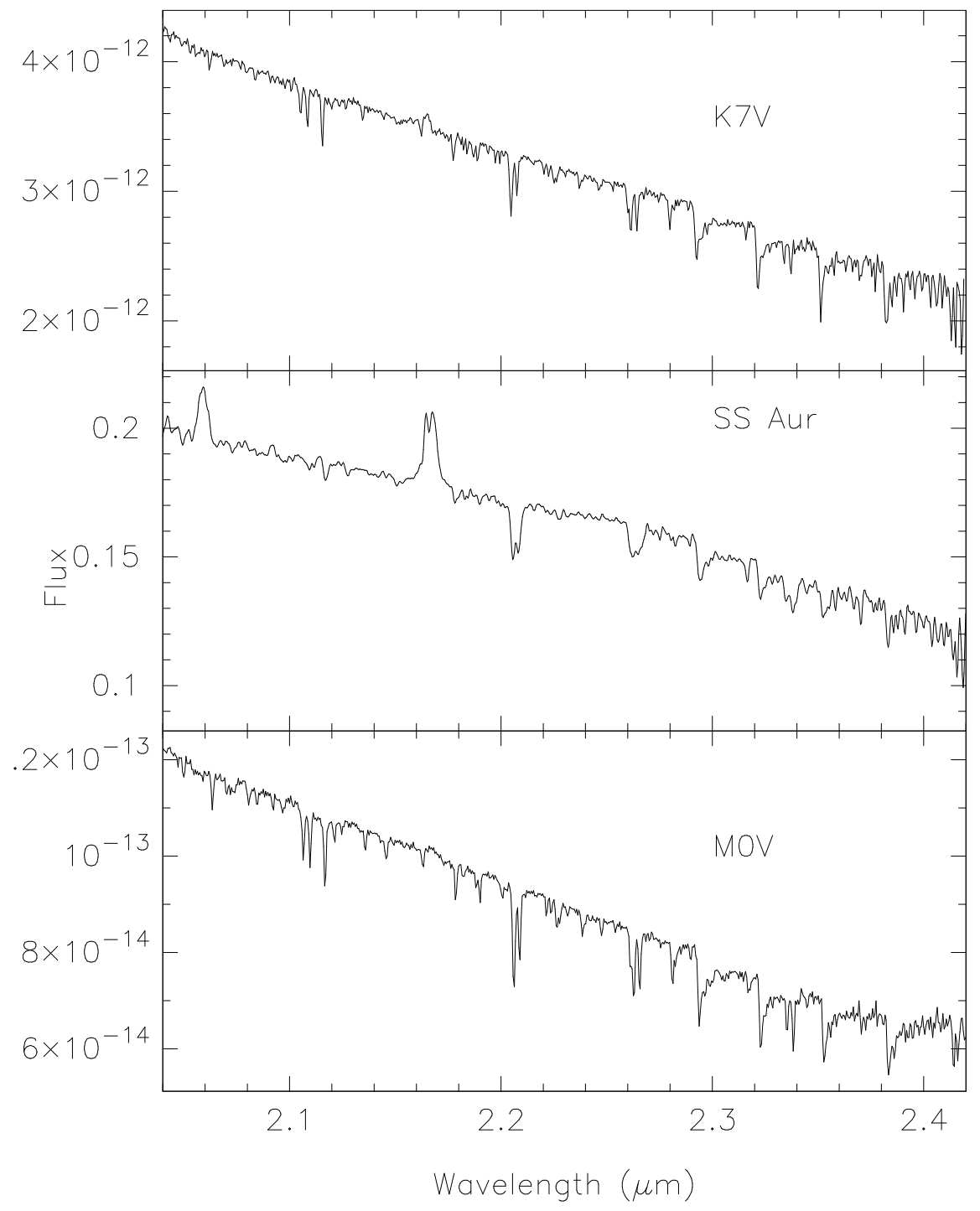

Fig. 5.- Infrared $K$-band spectrum of the dwarf nova SS Aur. The double-peaked Br $\gamma$ line is due to the accretion disk in this binary with an inclination of 38 degrees. 


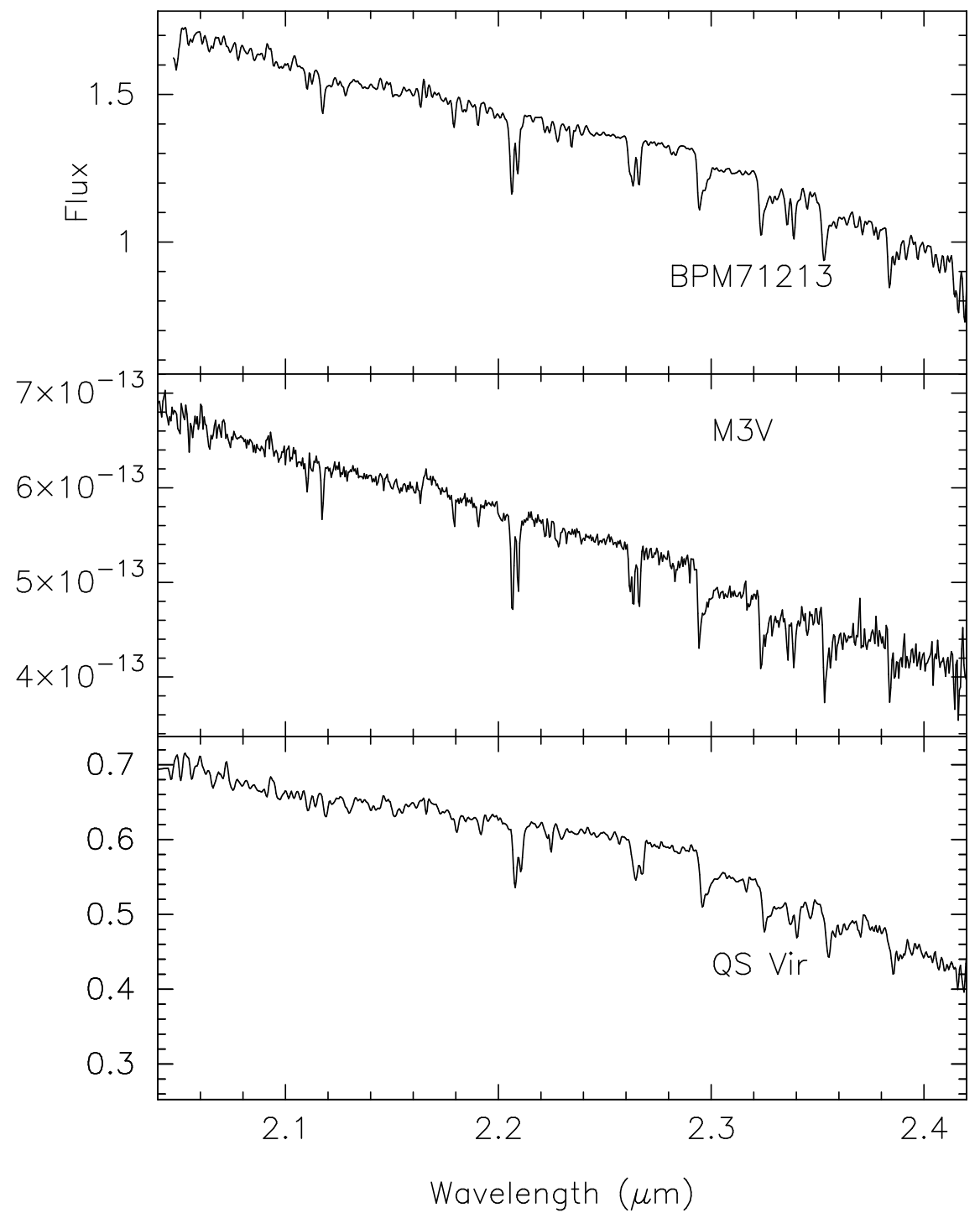

Fig. 6.- Infrared K-band spectra of the pre-CV BPM71213 and the hibernating CV QS Vir. Both systems have secondary stars near M2V-M4V and we show a single template spectrum of a M3V for comparison. Note that both stars show normal CO and other absorption bands. 


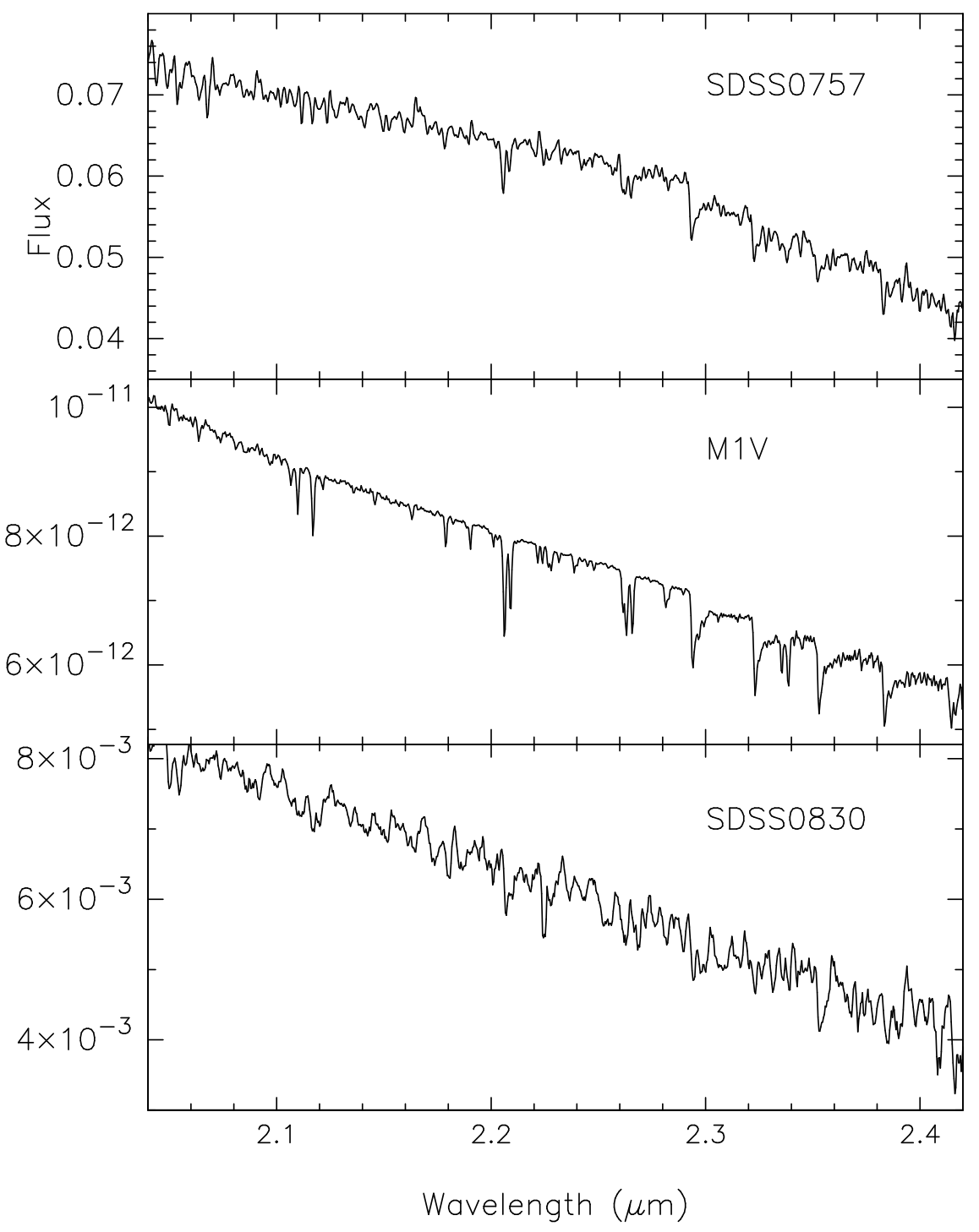

Fig. 7.- Infrared $K$-band spectra of the pre-CVs SDSS0757 and SDSS0830. Both systems have secondary stars near M0V-M2V and we show a single template spectrum of a M1V for comparison. Note that both stars show normal $\mathrm{CO}$ and other absorption bands. 


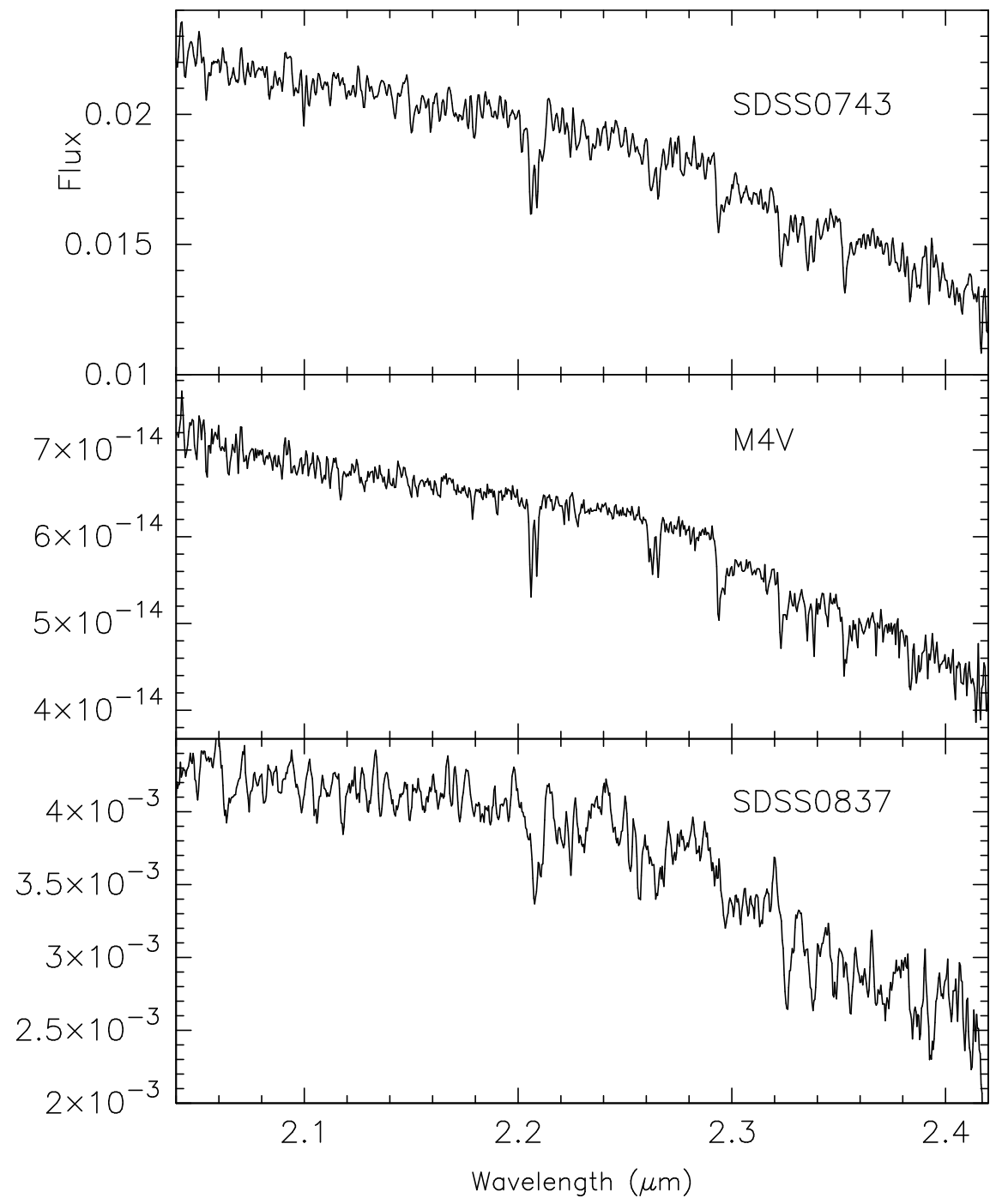

Fig. 8.- Infrared $K$-band spectra of the pre-CV SDSS0743 and the LARP (pre-polar) SDSS0837. Both systems have secondary stars near M4V and we show a single template spectrum of a M4V for comparison. 


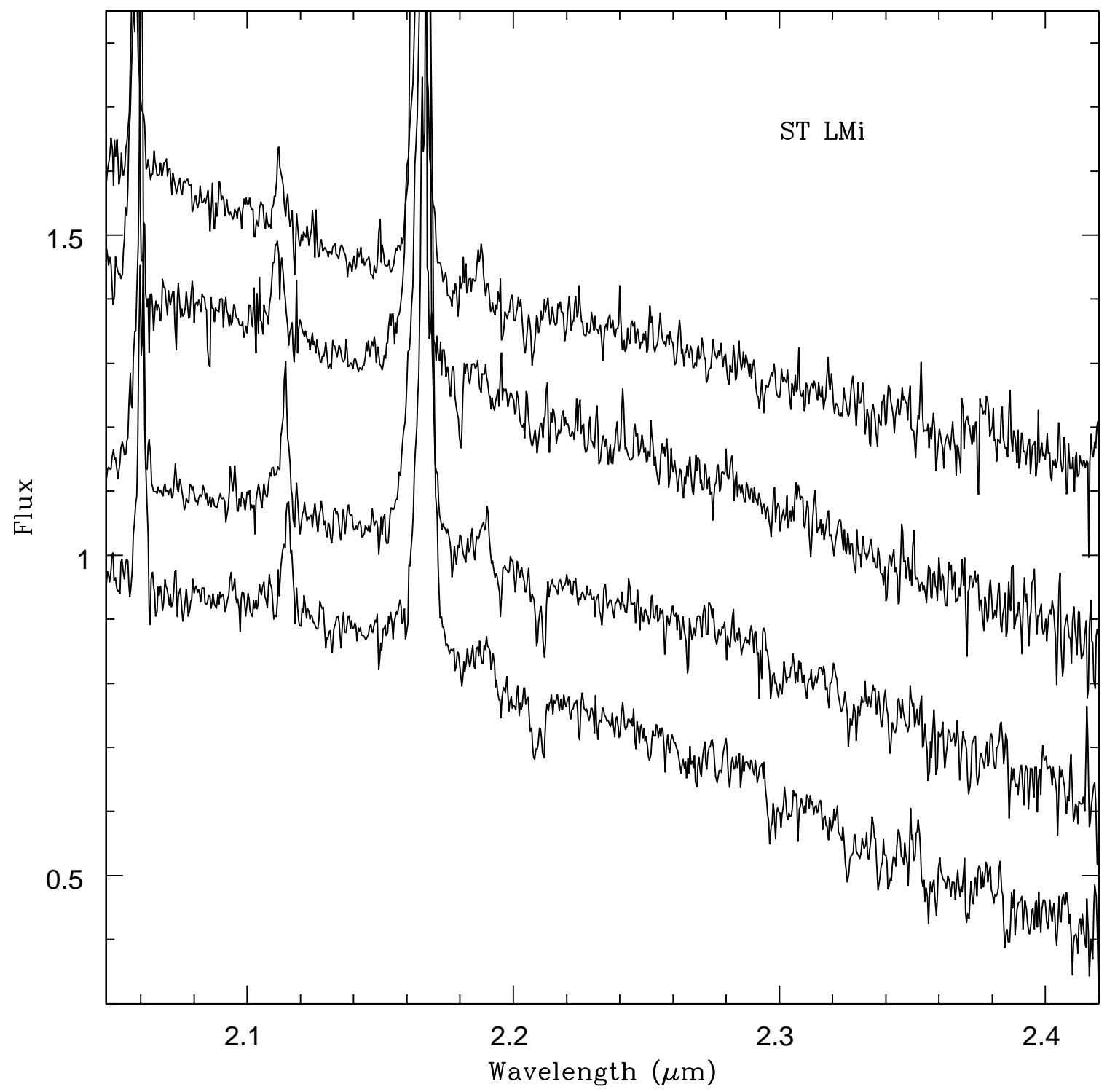

Fig. 9.- Four infrared $K$-band spectra of ST LMi covering a short segment (4X16 minutes) of its full orbital period $(1.09 \mathrm{hr})$. We note the short-term changes in the absorption lines and bands as evidenced by the Na doublet at 2.20/2.335 microns and the CO bands at 2.29 microns. 


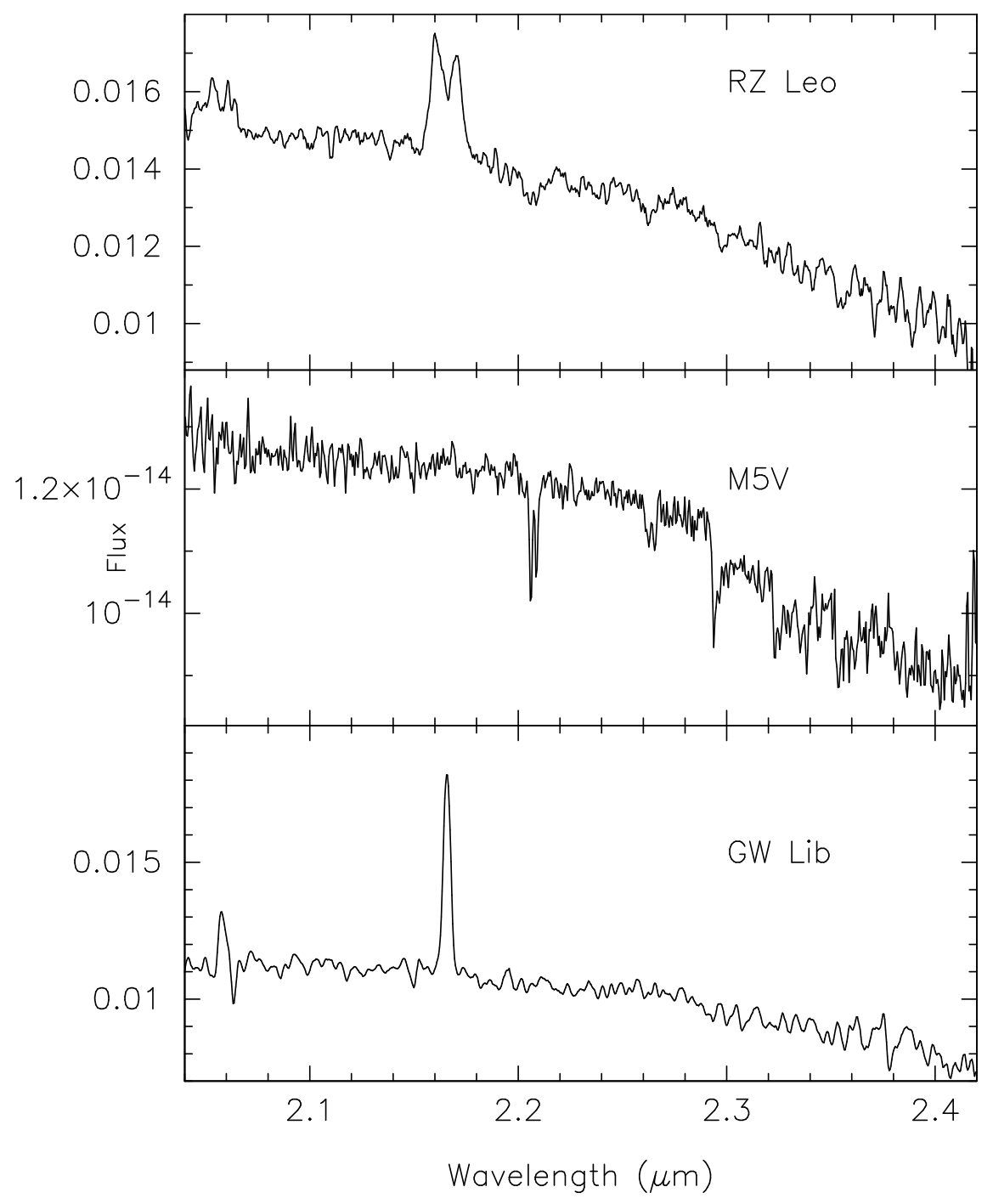

Fig. 10.- Infrared $K$-band spectra of the TOAD RZ Leo believed to have a M5V-like companion. Weak $\mathrm{CO}$ and other absorption lines are seen and we show a single star template spectrum of a M5V for comparison. The bottom spectrum is of the TOAD GW Lib showing string emission in $\operatorname{Br} \gamma$. We see no direct evidence of the secondary star, expected to be near M9V or a brown dwarf-like star, but do note the steam band/CO continuum break near 2.28 microns and redward. 


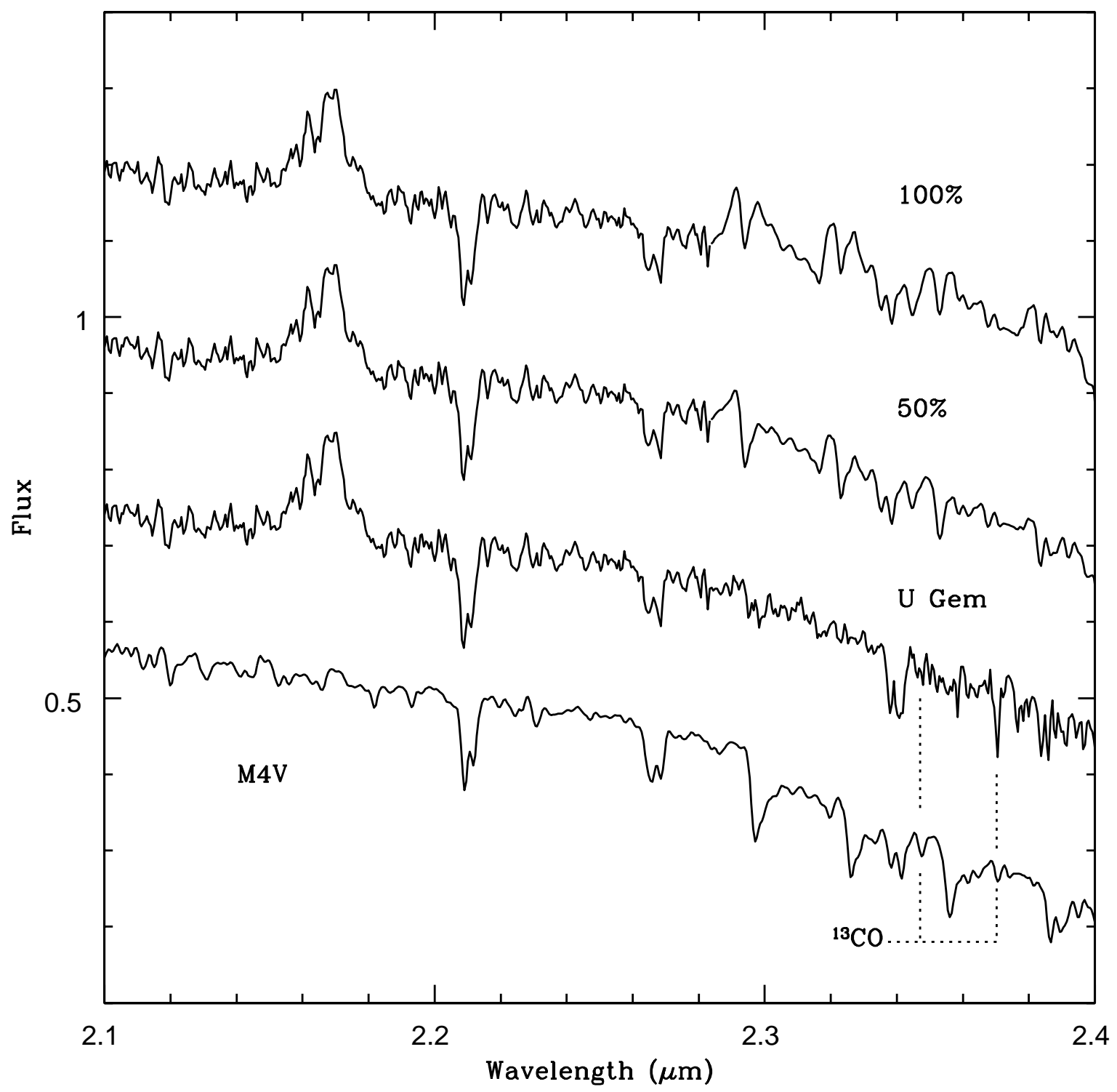

Fig. 11.- Model spectra used to assess CO emission contribution. Bottom spectrum is a single star template $\mathrm{M} 4 \mathrm{~V}$, the same spectral type as the secondary star in the dwarf nova $\mathrm{U}$ Gem. The observed $K$-band spectrum of $\mathrm{U}$ Gem is shown as the second from the bottom, revealing its very weak $\mathrm{CO}$ absorption but apparently normal $\mathrm{Na} \mathrm{I}$ and $\mathrm{Ca}$ I lines (see Harrison et al., 2005b). The top two spectra show our model M4V + CO emission spectrum combined with the observed U Gem spectrum. The model spectra show the choppy continuum and the trough created by the combination of the $\mathrm{CO}$ absorption from the secondary and the broader $\mathrm{CO}$ emission from the accretion disk. See text for details. 